\title{
Signatures of Heavy Vectors in Higgsless models
}

\author{
Jernej F. Kamenik* \\ INFN, Laboratori Nazionali di Frascati, I-00044 Frascati, Italy \\ J. Stefan Institute, 1000 Ljubljana, Slovenia \\ E-mail: jernej.kamenik@lnf.infn.it

\section{Oscar Catà} \\ INFN, Laboratori Nazionali di Frascati, I-00044 Frascati, Italy \\ E-mail: oscar.cata@lnf.infn.it

\section{Gino Isidori} \\ INFN, Laboratori Nazionali di Frascati, I-00044 Frascati, Italy \\ E-mail: gino.isidorielnf.infn.it
}

\begin{abstract}
One or more heavy spin-1 fields may replace the Higgs boson in keeping perturbative unitarity up to a few $\mathrm{TeV}$ while at the same time account for the electroweak precision tests. We study the Drell-Yan production of heavy vector and axial-vector states in generic Higgsless models at hadron colliders. We analyse in particular the $l^{+} l^{-}$, WZ, and three SM gauge boson final states. In the $l^{+} l^{-}$case we show how present Tevatron data restricts the allowed parameter space of these models. The two and three gauge boson final states (especially WZ, WWZ, and WZZ) are particularly interesting in view of the LHC, especially for light axial-vector masses, and could shed more light on the role of spin-1 resonances in the electroweak precision tests.
\end{abstract}

European Physical Society Europhysics Conference on High Energy Physics, EPS-HEP 2009,

July 16 - 222009

Krakow, Poland

${ }^{*}$ Speaker. 


\section{Motivation}

Experiments provide unambiguous indications that the SM gauge group is spontaneously broken $\left[S U(2)_{L} \times U(1)_{Y} \rightarrow U(1)_{Q}\right]$. One elementary $S U(2)_{L}$ scalar doublet with $\phi^{4}$ potential is the most economical and simple choice. However it is not the only allowed possibility. So far only the ground state of this Lagrangian has been tested with good accuracy through the mass measurements of $\mathrm{W}$ and $\mathrm{Z},\langle\phi\rangle \equiv v=246 \mathrm{GeV}$. Some dynamical sensitivity to the Higgs mechanism is obtained from electroweak precision observables (EWPO), which actually provide an indirect indication for a light Higgs, when the SM is regarded as an effective theory with a very high cut-off scale. But do we really need a fundamental Higgs field? EWPO indicate a spontaneous breaking of $S U(2)_{L} \times U(1)_{Y}$ where the breaking mechanism must respect, to a good accuracy, the custodial symmetry $\left[m_{Z}^{2} / m_{W}^{2} \simeq 1+\left(g^{\prime} / g\right)^{2}\right]$. General formulation of the symmetry breaking mechanism in absence of a fundamental Higgs (or for large Higgs masses) can be done in terms of a Chiral Lagrangian

$$
\mathscr{L}_{\chi}^{(2)}=\frac{v^{2}}{4} \operatorname{Tr}\left[D_{\mu} U^{\dagger} D^{\mu} U\right]
$$

where the field $U=\exp (i \Pi / v)$ containing the broken symmetry generators transforms under the global $S U(2)_{L} \times S U(2)_{R}$ symmetry as $U \rightarrow g_{R} U g_{L}^{\dagger}$, while its covariant derivate under the gauged $S U(2)_{L} \times U(1)_{Y}$ subgroup reads $D_{\mu} U=-i g^{\prime} B_{\mu} U+i g U W_{\mu}$. The spontaneous breaking of the global symmetry down to its vectorial part $S U(2)_{L+R}$ breaks the gauged subgroup down to $U(1)_{Q}$. The resulting EW chiral Lagrangian contains all the degrees of freedom we have directly probed in experiments. The naïve cut-off of the theory is dictated by the convergence of EW loops: $\Lambda_{N D A}=$ $4 \pi v \approx 3 \mathrm{TeV}$. It perfectly describes particle physics up $3 \mathrm{TeV}$ even beyond the tree level with only two drawbacks (which point towards the existence of new degrees of freedom below the naïve cut-off): (1) Violation of unitarity in longitudinal $W_{L} W_{L} \rightarrow W_{L} W_{L}$ scattering (tree-level amplitude violates unitarity for center-of-mass energy $\sqrt{s} \approx 1 \mathrm{TeV}$ ); (2)A bad fit to EWPO $\mathrm{S}$ and T [1].

\section{Introducing the Heavy Vectors}

A natural alternative to Higgs-type mechanisms in curing the problem of unitarity in $W W \rightarrow$ $W W$ scattering is represented by heavy vector fields. These are expected in many non-SUSY scenarios: techni-rho in technicolor or massive gauge bosons in 5-dimensional theories and hidden gauge models. The difficult task here is to cure at the same time unitarity and EWPO. It can be analysed in general terms by constructing an appropriate effective chiral Lagrangian with the heavy vector resonances $(\mathrm{R})$ as new explicit d.o.f.

$$
\mathscr{L}_{\chi}=\mathscr{L}_{\chi}^{(2)}+\mathscr{L}_{\text {kin }}\left(R, U, A_{i} ; m_{R}\right)+\mathscr{L}_{\text {int }}\left(R, U, A_{i} ; G_{R}, F_{R}, g_{R}\right),
$$

see ref. [2] for all parameter definitions and notation. We consider an effective theory based on the following two main assumptions [2]: (1) The (new) dynamics that breaks the SM EW symmetry is invariant under the global symmetry $S U(2)_{L} \times S U(2)_{R}$ and under the discrete parity P: $S U(2)_{L} \leftrightarrow$ $S U(2)_{R}$. (2) One vector $(\mathrm{V})$, or one vector + one axial-vector $(\mathrm{V}+\mathrm{A})$, both belonging to the adjoint representation of $S U(2)_{L+R}$ (triplets), are the only light fields below a cut-off $\Lambda=2-3 \mathrm{TeV}$. An 
effective Lagrangian expansion can now be performed for these vector fields based on ordering of operators according to the standard derivative (momentum) expansion.

The tree level unitarity of $W W$ scattering can be cured completely via the exchange of a single intermediate vector resonance provided the $V W_{L} W_{L}$ coupling $G_{V}$ takes on a particular value $G_{V}^{2}=$ $v^{2} / 3$. Requiring the theory to be unitary only below the naive cut-off, the unitarity constraint is almost insensitive to the value $m_{V}$ [2]. Also the leading contributions to $\mathrm{S} \& \mathrm{~T}$ are generated by the exchange of single heavy fields. It turns out there are two natural ways to accommodate the bounds. Either both $\mathrm{V}$ and are $\mathrm{A}$ light and almost degenerate, or only $\mathrm{V}$ is light, with a small $V W_{L} W_{T}$ coupling $F_{V}$. In both cases EWPO and unitarity can be accommodated for specific choices of the free parameters $\left(F_{A, V}, G_{V}\right)$. The main conclusion is however that we need at least one relatively light vector field [2].

\section{Production of Heavy Vectors at Hadron Colliders}

The main property of the vector fields is that the leading decay mode is into 2 longitudinal SM gauge bosons

$$
\Gamma_{V}^{+} \approx \Gamma_{W Z}^{V}=\frac{G_{V}^{2} m_{V}^{3}}{48 \pi v^{4}}\left[1+\mathscr{O}\left(g^{2} \varepsilon^{2}\right)\right], \quad \Gamma_{V}^{0} \approx \Gamma_{W W}^{V}=\Gamma_{W Z}^{V}\left[1+\mathscr{O}\left(g^{2} \varepsilon^{2}\right)\right],
$$

where $\varepsilon=v^{2} / m_{V}^{2}$, resulting in typically narrow widths of 5(40) GeV at heavy vector masses of $0.5(1.0) \mathrm{TeV}$. Note also that the $\mathrm{ZZ}$ channel is forbidden at tree level and that the coupling of heavy vectors to SM fermions is highly suppressed

$$
\operatorname{Br}\left(V^{0} \rightarrow q \bar{q}\right) \approx 3 \operatorname{Br}\left(V^{0} \rightarrow \ell^{+} \ell^{-}\right) \approx \frac{6 F_{V}^{2} m_{W}^{4}}{G_{V}^{2} m_{V}^{4}}
$$

which translates into $1.6(0.1) \%$ at heavy vector mass of $0.5(1.0) \mathrm{TeV}$. The main differences, when discussing axial resonances compared to vector ones, is that their decays to pairs of longitudinal SM gauge bosons are forbidden by parity so that depending on phase space the leading decay modes can be to pairs of heavy vectors and SM gauge fields (governed by an independent VAW coupling $g_{A}$ ). This in term leads to interesting phenomenology of three gauge boson final states.

The most general signature of Higgsless models is the appearance of the vector state in WW scattering $[p p \rightarrow V+j j(W W$ fusion $) \rightarrow W W(W Z)+j j]$. It constitutes a model-independent link with the unitarity problem. However, this requires a difficult analysis and high statistics[3]. A potentially cleaner signal (if the resonances are not too heavy) is the Drell-Yan production of the resonances and subsequent decay into $l^{+} l^{-}, 2$ and $3 \mathrm{SM}$ heavy gauge bosons [4]. These channels constitute a link to the contribution of the heavy vectors to EWPO. Given the narrow widths, for low masses the signals are quite large (see table 1$)$. However, the leading decay modes $(2 \mathrm{~W}, 3 \mathrm{~W})$ have low reconstruction efficiencies while the $l^{+} l^{-}$case is suppressed by the small $\mathrm{Br}\left(R \rightarrow l^{+} l^{-}\right)$.

The $l^{+} l^{-}$state of the art is the analysis of the $e^{+} e^{-}$(and di-muon) final states in $p-\bar{p}$ collisions published by $\mathrm{CDF}$ [5]. Using their $e^{+} e^{-}$data as normalization for the SM events (which takes into account all the relevant exp. efficiencies), we have produced an exclusion plot in the $F_{V}-m_{V}$ plane (see figure 4 in ref.[4]). The result is obtained under two main assumptions: $G_{V}$ is fixed by treelevel unitarity and $m_{A} \gg m_{V}$. Including the analysis of the di-muon channel removes all the areas, 


\begin{tabular}{|l|c|c|c|}
\hline & $M=500 \mathrm{GeV}$ & $M=750 \mathrm{GeV}$ & $M=1000 \mathrm{GeV}$ \\
\hline$\sigma\left(p p \rightarrow V^{+} \rightarrow X\right)_{\sqrt{s}=14 \mathrm{TeV}}$ & $11 \mathrm{pb}$ & $1.2 \mathrm{pb}$ & $0.23 \mathrm{pb}$ \\
\hline$\sigma\left(p p \rightarrow V^{+} \rightarrow X\right)_{\sqrt{s}=10 \mathrm{TeV}}$ & $6.7 \mathrm{pb}$ & $0.7 \mathrm{pb}$ & $0.13 \mathrm{pb}$ \\
\hline$\sigma\left(p p \rightarrow V^{+} \rightarrow X\right)_{\sqrt{s}=7 \mathrm{TeV}}$ & $4.2 \mathrm{pb}$ & $0.32 \mathrm{pb}$ & $0.06 \mathrm{pb}$ \\
\hline
\end{tabular}

Table 1: Summary of the leading-order cross sections for the production of a light charged vector resonance in $p p$ collisions at $\sqrt{s}=14 \mathrm{TeV}, \sqrt{s}=10 \mathrm{TeV}$ and $\sqrt{s}=7 \mathrm{TeV}$. The results are obtained summing over all decay products in the mass range $\left|M_{X}-M_{V}\right| \leq 3 \Gamma_{V}$, setting $F_{V}=2 G_{V}$, and fixing $G_{V}$ from unitarity $\left(G_{V}=v / \sqrt{3}\right)$. The results for different values of $F_{V}$ can be obtained scaling the figures in the table by $F_{V}^{2} /\left(2 G_{V}\right)^{2}$.

where an excess is seen in the electron-positron data. If, on the other hand, an excess at higher mass will become significant, we can hope to see a clear signal at the LHC (even with $1-2 \mathrm{fb}^{-1}$ ). No huge peaks as with a sequential Z' are expected, but they should be clearly visible [4].

More prospective are two and three SM gauge boson final states. Some illustrative examples are shown on figures $6-8$ in ref.[4]), where we have used $F_{V}=2 G_{V}, F_{A}=F_{V}$ and $G_{V}$ is fixed by unitarity, while $g_{A}=1 / 2$. Note that the expected reconstructed signal will necessarily be further suppressed by typically low reconstruction efficiencies, e.g. for the purely leptonic channels $[W Z] B r Z_{\text {lept }} \times B r W_{\text {lept }}=1.5 \%$ while for $[W W Z] B r Z_{\text {lept }} \times B r W_{\text {lept }} \times B r W_{\text {had }}=0.9 \%$. Due to the typically sizable contribution of the intermediate vector resonance in the WWZ final state it is also worth looking at the WZ invariant- mass distribution.

\section{Conclusions}

Heavy vector fields, which replace the Higgs boson in maintaining perturbative unitarity up to LHC energies, are naturally expected in a wide class of Higgsless models. The most general signature of these models is the appearance of the lightest vector state in WW scattering (modelindependent link with the unitarity problem). The Drell-Yan production of the new states is subject to larger uncertainties. For light $m_{V(A)}$ we could expect visible signals (even with low statistics), and the information could help to clarify the role of the heavy vectors in EWPO. The results in the $e^{+} e^{-}$channel from Tevatron are already providing significant information, while the 2 and $3 \mathrm{SM}$ gauge boson final states seem to be quite promising and would deserve a more realistic study.

\section{References}

[1] M. E. Peskin and T. Takeuchi, Phys. Rev. Lett. 65, 964 (1990); G. Altarelli and R. Barbieri, Phys. Lett. B 253, 161 (1991).

[2] R. Barbieri, G. Isidori, V. S. Rychkov and E. Trincherini, Phys. Rev. D 78, 036012 (2008).

[3] A. Belyaev, arXiv:0711.1919 [hep-ph].

[4] O. Cata, G. Isidori and J. F. Kamenik, Nucl. Phys. B 822, 230-244 (2009)

[5] T. Aaltonen et al. [CDF Collaboration], Phys. Rev. Lett. 102, 031801 (2009); ibid. 102, 091805 (2009) 